\title{
Multiple Attribute Decision-making with Interval Numbers Projection and Its Application in the Power grid Planning
}

\author{
Feng Ren \\ School of Business and Administration, North China Electric Power University, Baoding 071003, Hebei Province, China
}

\begin{abstract}
In this paper, the theory of interval numbers was applied into multiple attribute decision-making to overcome the limitation of a single real number for attribute value. The interval positive ideal point of the decision problem was constructed based on the interval numbers of the attributes. The alternatives were projected to the positive ideal point according to the projection theory, and then the optimal closeness can be obtained with which the optimal solution can be chosen. Applying this method to the optimization of power grid planning, the example analysis shows that the method is scientific and practical.
\end{abstract}

Keywords-multiple attribute decision-making; interval numbers; projection pursuit; power grid planning.

\section{INTRODUCTION}

Multiple attribute decision making is becoming an immortal research focus in recent years in the academic circles. Multiple attribute decision-making with multiple goal decision constituting the multi-criteria decisionmaking system is the principal branch of operations research and management science. Many scholars engaged in the study of the theory of multiple attribute decision making, usually with AHP, fuzzy set theory and TOPSIS method, combined weights theory and methods[1-7], and its range of application is in risk assessment and prediction, etc[8-9].

However, attribute values with an interval number rather than a numerical in a multiple attribute decision can be often more convincing, and the decision results also have more practical application value. In this paper, we assigned interval numbers to the values of attributes, obtained the interval positive ideal point of the decision problem, constructed the interval projection multiple attribute decision-making method, and applied it to the power grid planning optimization. The rest of this article is arranged as following: the second section presents the theoretical basis of the study; the third section is the description of the modeling process; the fourth section has carried on the empirical analysis; and the fifth section is the conclusion.

\section{METHODOLOGY}

Definition 1: Set weighted normalized decision matrix $\tilde{Y}=\left(\tilde{y}_{i j}\right)_{n \times m}$, call $\tilde{y}^{+}=\left(\tilde{y}_{1}^{+}, \tilde{y}_{2}^{+}, \cdots, \tilde{y}_{m}^{+}\right)$interval positive ideal point, where $\tilde{y}_{i j}=\left[y_{i j}^{L}, y_{i j}^{U}\right], \tilde{y}_{i j}=\omega_{j} \tilde{r}_{i j}, i \in N, j \in M$, $\tilde{y}_{j}^{+}=\left[y_{j}^{+^{L}}, y_{j}^{+^{U}}\right]=\left[\max _{i}\left(y_{i j}^{L}\right), \max _{i}\left(y_{i j}^{U}\right)\right], j \in M$.

Definition 2: set $\alpha=\left(\alpha_{1}, \alpha_{2}, \cdots, \alpha_{m}\right)$, $\beta=\left(\beta_{1}, \beta_{2}, \cdots, \beta_{m}\right)$ as two vectors, define

$$
P R J_{\beta}(\alpha)=\frac{\sum_{j=1}^{m} \alpha_{j} \beta_{j}}{\sqrt{\sum_{j=1}^{m} \beta_{j}^{2}}}
$$

as the projection of $\alpha$ on $\beta$. In general, the bigger the value of $P R J_{\beta}(\alpha)$, the closer these two vectors are. Similarly,

$$
P R J_{\tilde{y}^{+}}\left(\tilde{y}_{i}\right)=\frac{\sum_{j=1}^{m}\left[\tilde{y}_{i j}^{L} y_{j}^{+L}+\tilde{y}_{i j}^{U} y_{j}^{+U}\right]}{\sqrt{\sum_{j=1}^{m}\left[\left(y_{j}^{+L}\right)^{2}+\left(y_{j}^{+U}\right)^{2}\right]}}
$$

where $\tilde{y}_{i}=\left(\tilde{y}_{i 1}, \tilde{y}_{i 2}, \cdots, \tilde{y}_{i m}\right), i \in N$. Obviously, the bigger the value of $P R J_{\tilde{y}^{+}}\left(\tilde{y}_{i}\right)$, the closer the alternative $x_{i}$ and the interval positive ideal point are, that is to say, the better the alternative $x_{i}$ is.

\section{MODELLING PROCESS}

The steps of interval projection multiple attribute decision-making method are as follows.

Step1, measuring $x_{i}$ according to attribute $u_{j}$, we can get the attribute values $\tilde{a}_{i j}\left(\tilde{a}_{i j}=\left[a_{i j}^{L}, a_{i j}^{U}\right]\right)$, which constitute the decision matrix $\tilde{A}=\left(\tilde{a}_{i j}\right)_{n \times m}$. There are two kinds of the most common attribute types: the efficiency type and the cost type. Let $I_{j}(j=1,2)$ denote the index sets of the efficiency type and the cost type, respectively. In order to eliminate the influence of different physical dimension to the decision result, we can use the following 
formulas to transform the decision matrix $\tilde{A}$ into normalized matrix $\tilde{R}=\left(\tilde{r}_{i j}\right)_{n \times m}, \tilde{r}_{i j}=\left[r_{i j}^{L}, r_{i j}^{U}\right]$,

$$
\begin{gathered}
\tilde{r}_{i j}=\tilde{a}_{i j} /\left\|\tilde{a}_{j}\right\|, i \in N, j \in I_{1} \\
\tilde{r}_{i j}=\left(1 / \tilde{a}_{i j}\right) /\left(\left\|1 / \tilde{a}_{j}\right\|\right), i \in N, j \in I_{2} \\
\text { where }\left\|\tilde{a}_{j}\right\|=\sqrt{\sum_{i=1}^{n} \tilde{a}_{i j}^{2},\left\|1 / \tilde{a}_{j}\right\|=\sqrt{\sum_{i=1}^{n}\left(1 / \tilde{a}_{i j}\right)^{2}} .}
\end{gathered}
$$

According to interval algorithms, the formulas (3) and (4) can be written as the following forms:

$$
\begin{aligned}
& \left\{\begin{array}{l}
r_{i j}^{L}=a_{i j}^{L} / \sqrt{\sum_{i=1}^{n}\left(a_{i j}^{U}\right)^{2}}, i \in N, j \in I_{1} \\
r_{i j}^{U}=a_{i j}^{U} / \sqrt{\sum_{i=1}^{n}\left(a_{i j}^{L}\right)^{2}}
\end{array}\right. \\
& \left\{\begin{array}{l}
r_{i j}^{L}=\left(1 / a_{i j}^{U}\right) / \sqrt{\sum_{i=1}^{n}\left(1 / a_{i j}^{L}\right)^{2}}, i \in N, j \in I_{2} \\
r_{i j}^{U}=\left(1 / a_{i j}^{L}\right) / \sqrt{\sum_{i=1}^{n}\left(1 / a_{i j}^{U}\right)^{2}}
\end{array}\right.
\end{aligned}
$$

Step2, use WAA operator to compile the attributes of the alternatives $x_{i}(i \in N)$, and obtain the comprehensive attribute values $\tilde{y}_{i}(\omega)(i \in N)$ :

$$
\tilde{y}_{i}(\omega)=\sum_{j=1}^{m} \omega_{j} \tilde{r}_{i j}
$$

Then, we can get the weighted normalized decision matrix $\tilde{Y}=\left(\tilde{y}_{i j}\right)_{n \times m}$.

Step3, determine the interval type positive ideal point $\tilde{y}^{+}$.

Step4, find the projection of $x_{i}$ on the interval ideal point: $P R J_{\tilde{y}^{+}}\left(\tilde{y}_{i}\right), i \in N$.

Step5, according to the values of $P R J_{\tilde{y}^{+}}\left(\tilde{y}_{i}\right), i \in N$, sort the alternatives and select the biggest one as the optimal decision.

\section{COMPUTATIONAL EXAMPLE}

Power grid planning is a typical multiple attribute decision-making problem, in which many factors should be considered. A regional power grid construction has three alternatives $x_{i}(i=1,2,3)$. The attributes need to be considered are project costs $\left(u_{1}\right)$, life cycle $\left(u_{2}\right)$, average duration $\left(u_{3}\right)$, availability of land $\left(u_{4}\right)$ and reliability $\left(u_{5}\right)$. The decision matrix $\tilde{A}$ is shown as Table 1 .

According to the earlier studies of research group, the attribute weight vector $\omega=(0.2189,0.2182,0.1725,0.2143,0.1761)$ is obtained by AHP method.

Step1, according to formulas (5) and (6), transform decision matrix $\widetilde{A}$ into normalized decision matrix $\widetilde{R}$, and the result is shown as Table2.

Step2, Use the attribute weight vector $\omega$ and normalized decision matrix $\widetilde{R}$ to construct weighted normalized decision matrix $\tilde{Y}$, and the result is shown as Table3.

TABLE I. DECISION MATRIX

\begin{tabular}{c|ccccc}
\hline & $u_{1}$ & $u_{2}$ & $u_{3}$ & $u_{4}$ & $u_{5}$ \\
\hline$x_{1}$ & {$[58.9,59.0]$} & {$[200,250]$} & {$[1.9,2.1]$} & {$[0.990,0.991]$} & {$[0.907,0.909]$} \\
$x_{2}$ & {$[58.5,58.7]$} & {$[340,350]$} & {$[3.4,3.5]$} & {$[0.990,0.992]$} & {$[0.910,0.912]$} \\
$x_{3}$ & {$[58.0,58.5]$} & {$[290,310]$} & {$[2.0,2.2]$} & {$[0.992,0.993]$} & {$[0.914,0.917]$} \\
\hline
\end{tabular}

TABLE II. NORMALIZED DECISION MATRIX

\begin{tabular}{c|ccccc}
\hline & $u_{1}$ & $u_{2}$ & $u_{3}$ & $u_{4}$ & $u_{5}$ \\
\hline$x_{1}$ & {$[0.5721,0.5757]$} & {$[0.3772,0.5106]$} & {$[0.6080,0.1265]$} & {$[0.5762,0.5775]$} & {$[0.5738,0.5765]$} \\
$x_{2}$ & {$[0.5750,0.5796]$} & {$[0.6413,0.7149]$} & {$[0.3648,0.4098]$} & {$[0.5762,0.5781]$} & {$[0.5757,0.5784]$} \\
$x_{3}$ & {$[0.5770,0.5846]$} & {$[0.5470,0.6332]$} & {$[0.5803,0.6967]$} & {$[0.5774,0.5787]$} & {$[0.5782,0.5816]$} \\
\hline
\end{tabular}

TABLE III. WEIGHTED NORMALIZED DECISION MATRIX

\begin{tabular}{c|ccccc}
\hline & $u_{1}$ & $u_{2}$ & $u_{3}$ & $u_{4}$ & \\
\hline$x_{1}$ & {$[0.1252,0.1260]$} & {$[0.0823,0.1114]$} & {$[0.1049,0.1265]$} & {$[0.1235,0.1238]$} & {$[0.1010,0.1015]$} \\
$x_{2}$ & {$[0.1259,0.1269]$} & {$[0.1399,0.1560]$} & {$[0.0629,0.0707]$} & {$[0.1235,0.1239]$} & {$[0.1014,0.1019]$} \\
$x_{3}$ & {$[0.1263,0.1280]$} & {$[0.1194,0.1382]$} & {$[0.1001,0.1202]$} & {$[0.1237,0.1240]$} & {$[0.1018,0.1024]$} \\
\hline
\end{tabular}


Step3, Calculate the interval positive ideal point according to the definition1:

$\tilde{y}^{+}=([0.1263,0.1280],[0.1399,0.1560],[0.1049,0.1265],[0.1237,0.1240],[0.1018,0.1024])$

Step4, according to formula (2), calculate the projection of alternative $x_{i}$ on the interval positive ideal point:

$$
\begin{aligned}
& P R J_{\tilde{y}^{+}}\left(\tilde{y}_{1}\right)=0.3537 \quad, \quad P R J_{\tilde{y}^{+}}\left(\tilde{y}_{2}\right)=0.2717, \\
& P R J_{\tilde{y}^{+}}\left(\tilde{y}_{3}\right)=0.3758 .
\end{aligned}
$$

Step5, according to the values of $P R J_{\tilde{y}^{+}}\left(\tilde{y}_{i}\right)(i=1,2,3)$, the alternatives are sorted as $x_{3} \succ x_{1} \succ x_{2}$. So, we can draw a conclusion that $x_{i}$ is the optimal planning.

\section{V.CONCLUSIONS}

Multiple attribute decision-making method has wide basis, and its application has broad prospects. The scientific nature of the attribute value is the basis of the reasonability for decision-making results. In this research, the attribute values were regarded as interval numbers, which is more in line with the nature of things. Interval positive ideal point makes the decision problems have a scientific reference standard. Interval projection multiple attribute decisionmaking method was applied to power grid planning, and the results showed that the method is feasible and has certain applicability.

\section{ACKNOWLEDGEMENTS}

The research work was supported by the Fundamental Research Funds for the Central Universities(12MS136) and the Soft Science of Hebei Province（12457301）

\section{REFERENCES}

[1] Kuang Tanli. Study on Multi-attribute Decision Making Method Based on Fuzzy Soft Sets [D]. Chongqing University, 2013

[2] Li Xihua. Intuitionistic Fuzzy Multiple Attribute Complex-Large Group Decision Making Method Based on Prospec Theory [D]. Central South University, 2012

[3] Zhou Ya. The Study of TOPSIS Method in Multiple Attribute Decision Making [D]. Wuhan University of Technology, 2009

[4] Du Lina. Multi-attribute Decision Making by Analytic Hierarchy Process [J]. Journal of Gansu Lianhe University (Natural Sciences). 2007(05): 31-35

[5] You Tianhui, Fan Zhiping. An Error Propagation Method for Assessing Entropy Weights in Uncertain Multiple Attribute Decision Making [J]. Systems Engineering, 2003(01): 101-104

[6] Chen Huayou. Research on Optimal Combination Determining Weights Method for Multiple Attribute Decision Making [J]. Operations Research and Management Science, 2003(02): 6-10

[7] Wang Zhongxing, Mu Xiong, Li Qiaoxing. A New Combination Weighting Method in Multiple Attribute Decision Making [J]. Communication on Applied Mathematics and Computation, 2003(02): $55-62$

[8] Liu Jinpei. Multi-attribute Decision Making and Time Series Forecast Based on Aggregation Operators [D]. Tianjin University, 2012

[9] Yang Xiaoling. Research on the Application of MADM in Flood Risk Evaluation [D]. Huazhong University of Science and Technology, 2012 